\title{
Carbon Ion Radiotherapy in the Management of the Tumors of the Peripheral Nervous System
}

\author{
VIVIANA VITOLO ${ }^{1}$, MARIA ROSARIA FIORE ${ }^{1}$, AMELIA BARCELLINI ${ }^{1}$, BARBARA VISCHIONI ${ }^{1}$, \\ ALBERTO IANNALFI ${ }^{1}$, ANGELICA FACOETTI $^{1}$, PIERO FOSSATI ${ }^{1}$, MARIA BONORA $^{1}$, \\ SARA RONCHI ${ }^{1}$, EMMA D'IPPOLITO ${ }^{1}$, RACHELE PETRUCCI ${ }^{1}$, GISELA VISELNER ${ }^{1}$, \\ MARIO CIOCCA ${ }^{1}$, LORENZO PREDA $^{1,2}$, FRANCESCA VALVO $^{1}$ and ROBERTO ORECCHIA ${ }^{1,3}$ \\ ${ }^{1}$ National Center of Oncological Hadrontherapy (Fondazione CNAO), Pavia, Italy; \\ ${ }^{2}$ Department of Clinical-Surgical, Diagnostic and Pediatric Sciences, University of Pavia, Pavia, Italy; \\ ${ }^{3}$ European Institute of Oncology, Milan, Italy
}

\begin{abstract}
Background/Aim: Malignant peripheral nerve sheaths tumors (MPNST) are rare, highly aggressive and radioresistant tumors of soft tissue. Carbon ion radiotherapy (CIRT) seems to be an attractive treatment option when surgery is not possible or refused by the patient. The aim of this study was to report our preliminary results (outcomes and toxicity) of CIRT in the treatment of MPNSTs. Patients and Methods: Our study retrospectively analyzed 13 patients (median age $=54$ years; range $=23-72$ years) with MPNST treated with CIRT for a median total dose of $73.6 \mathrm{~Gy}$ (relative biological effectiveness) (range $=70.4-76.8 \mathrm{~Gy}$ ) between 2013 and 2016. Results: Six local progressions were recorded, with a median local progression-free survival of 23.2 months (range=2.2-45.4 months). Acute toxicities were mild. Late toxicity of grade 2 or more was noted in two patients. Conclusion: CIRT yielded good local control with a low rate of acute and late toxicities.
\end{abstract}

Malignant peripheral nerve sheath tumors (MPNSTs) are rare and highly aggressive soft-tissue tumors that arise from cellular components found in the sheath of peripheral nerves or from pre-existing benign tumors. MPNSTs are characterized by rapid, infiltrating growth and hematogenous dissemination. The reported incidence is 1: 1,000,000 per years, representing 5-10\% of all malignant soft-tissue tumors. They occur mainly in adults, with only $10-20 \%$ of MPNSTs being diagnosed in the first two decades of life. MPNST can arise in three settings: Sporadic, post-irradiation, and associated with neurofibromatosis type 1 .

Correspondence to: Angelica Facoetti, Fondazione CNAO, Strada Campeggi 53, Pavia, Italy. Tel: +39 0382078455, Fax: +39 +39 0382078903, e-mail: Facoetti@cnao.it

Key Words: Tumors of the peripheral nervous system, MPNST, carbon ion radiotherapy, radiation therapy.
They usually present as a mass associated with pain or neurological symptoms (1-3).

Because of the rarity of this tumor type, there are few published reports and little information is available on its clinical management. Surgery with negative margins is the mainstay of treatment but frequently is not free from potentially unacceptable morbidity due to the inherent necessity to sacrifice the involved nerves. Primary disease presents problems in terms of local control (LC) even after microscopic complete resection. In this favorable setting, the local recurrence rate is $20-38 \%$ but increases in cases of negative prognostic factors such as positive margins, gross residual disease, recurrence, and head and neck as initial presentation site $(4,5)$.

Radiation therapy (RT) is the standard of care in adjuvant setting, but radical RT can be an alternative in case of unresectable tumors or when a patient refuses destructive surgery. To obtain long-term LC in patients with MPNST, high doses with conventional RT are required but, unfortunately, these doses would entail high morbidity. For their intrinsic better characteristics of dose distribution, and biological effectiveness, carbon ion RT (CIRT) may be promising for treatment of MPNSTs $(6,7)$.

The aim of the present article is to report our early experience with CIRT in the treatment of MPNSTs.

\section{Patients and Methods}

The medical records of 13 consecutive patients with MPNSTs treated with CIRT at our Institution between 2013 and 2016 were reviewed. Diagnosis was histologically confirmed in all patients and all had macroscopic disease. Initial work-up included at least clinical examination, magnetic resonance imaging (MRI) of the primary lesion and computed tomography (CT) of the chest and abdomen. Patients were immobilized with a thermoplastic mask and a custom polystyrene bead pillow in supine or prone position, based on the tumor site. Treatment planning was based on CT and contrast-enhanced MRI rigidly registered. 
The target volumes were outlined as follow: Gross tumour volume (GTV) included all visible gross disease; clinical target volume (CTV) was GTV with a margin of between 3 to $10 \mathrm{~mm}$, based on the anatomical location; planning target volume (PTV) included CTV plus 3 to $5 \mathrm{~mm}$ margins. Margins were reduced at anatomical borders or to spare directly adjacent radiosensitive organs at risk at the discretion of the radiation oncologist. Treatment planning was performed using Syngo RT Planning TPS, version C13 (Siemens AG Healthcare, Erlangen, Germany), and the biological effectiveness of the particle beam was incorporated into the planning software according to the Local Effect Model version $\mathrm{I}$, with the following parameters $\left(\alpha_{\gamma} 0.1 \mathrm{~Gy}^{-1}, \beta_{\gamma} 0.05 \mathrm{~Gy}^{-2}\right.$, Dt 30 $\mathrm{Gy}$, nuclear radius $5 \mu \mathrm{m})$. Intensity-modulated particle therapy was employed for optimization.

Examples of three-dimensional dose distributions for patients with tumors are shown in Figures 1 and 2.

Regular follow-up took place at our Institution, including clinical examination with scoring of toxicity according Common Terminology Criteria for Adverse Events (CTCAE) Version 4.0 (8) and MRI of the primary lesion every 3 months for the first 2 years and every 6 months thereafter; chest and abdomen CT were performed at least every 6 months. Additional tests or imaging modalities were performed to confirm or exclude disease progression at the discretion of the radiation oncologist.

Continuous variables are summarized as medians, means, standard deviations (SD) and ranges. Categorical variables are listed as frequencies and proportions. Time to event data were calculated from the end of CIRT treatment to last follow-up or death, according to the Kaplan-Meier method. LC was defined as no evidence of tumor regrowth in the PTV or absence of further tumor regrowth after best response of the treated lesion. Progression-free survival was defined as the absence of locoregional or distant failure. Overall survival was defined as the time from CIRT to death from any cause

\section{Results}

Patient and tumor characteristics and treatment details are summarized in Table I.

Overall, the median age was 54 years (range $=23-72$ years). Six patients were male and seven female. MPNST was associated with neurofibromatosis type 1 in two patients and with a post irradiation status in one patient (previously irradiated for non-Hodgkin's lymphoma). At the time of diagnosis, all patients had been surgically treated by partial resection leading to microscopic positive margins in five patients and macroscopic positive margins in six cases, while three patients underwent macroscopic gross resection without formal evaluation of resection margins in the histological report. Five patients had received previous chemotherapy: two with adriamycin/ifosfamide, one with epirubicine/ ifosfamide, and two with epirubicine/ifosfamide followed by etoposide/ifosfamide, but no chemotherapy was applied concurrently with CIRT. Eight patients presented at our Center with recurrent disease.

All patients received CIRT, and a median dose of $73.6 \mathrm{~Gy}$ [relative biological effectiveness (RBE)] in 16 fractions
Table I. Demographics, tumor characteristics and treatment details.

\begin{tabular}{lc}
\hline Characteristic & Value \\
\hline Number of patients & 13 \\
Median age \pm SD (years) & $54 \pm 14.8$ \\
Gender, $\mathrm{n}$ & \\
$\quad$ Male & 6 \\
Female & 7 \\
Tumor location, $\mathrm{n}$ & \\
Head and neck & 6 \\
Brachial plexus & 4 \\
Trunk & 2 \\
$\quad$ Lumbar plexus & 1 \\
Recurrent disease, $\mathrm{n}$ & \\
$\quad$ Yes & 8 \\
$\quad$ No & 5 \\
Median overall treatment time \pm SD (days) & $27 \pm 2.9$ \\
Median total dose \pm SD $($ Gy RBE) & $73.6 \pm 3.69$ \\
Median fraction dose \pm SD (Gy RBE) & $4.6 \pm 0.23$ \\
\hline
\end{tabular}

RBE: Relative biological effectiveness; SD: standard deviation.

(range=70.4-76.8 Gy RBE) to the high-risk volume delivered with a median daily dose of $4.6 \mathrm{~Gy}$ (RBE), four fractions per week. Treatment was well tolerated and no interruption was needed. Maximum intra-treatment-related RT toxicity was grade 2 erythema in two patients. Acute toxicities were mild, no side effects of more than grade 2 were observed and no treatment interruption was needed. Late toxicity of grade 2 or more was found in two patients: brain radionecrosis successfully treated with high dose corticosteroids in one, and, not unexpectedly, brachial neuropathy (in MPNST involving the brachial plexus) in another. Overall, after a median follow-up of 24.6 months (range=2.2-45.4 months) and a median local progression-free survival of 23.2 months (range=2.2-45.4 months), six patients had experienced local progression. Three patients developed distant failure and five had died from their disease. The 2-year LC and 2-year overall survival rates were $63 \%$ and $60 \%$, respectively (Figure 3 ).

\section{Discussion}

Local control of MPNST and survival of patients appear to be related to complete tumor resection and every effort should be made to perform an excision with tumor-free histological margins. However, even after optimal surgery with wide margins, the local recurrence rate remains high (20-38\%) and it is not rare to see relapses along the nerve, not in continuity with the tumor bed. Moreover, surgery entails potential morbidity due to the typical growth of MPNSTs from the major nerve plexuses or because lesions involve large nerves or are so large that amputation is necessary (1-4). 


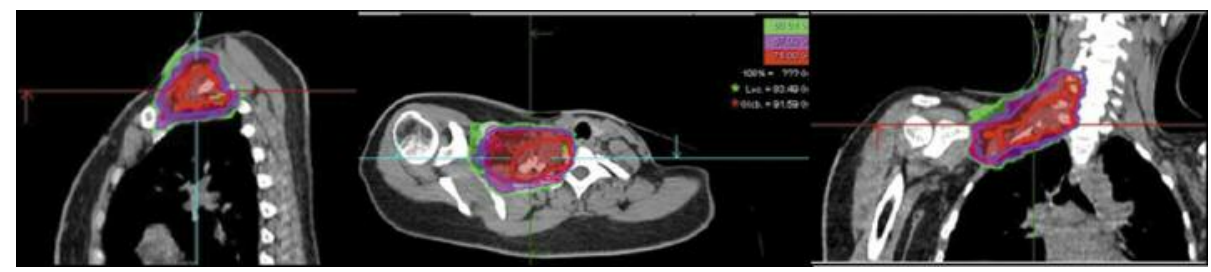

Figure 1. Example of dose distribution using carbon ion radiotherapy in a patient with malignant peripheral nerve sheaths tumors of the brachial plexus.

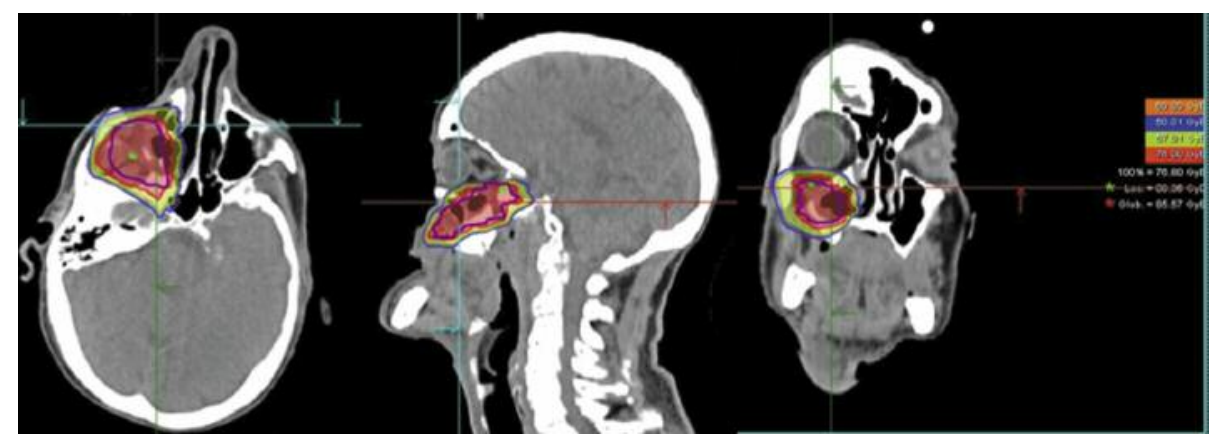

Figure 2. Example of dose distribution using carbon ion radiotherapy in a patient with malignant peripheral nerve sheaths tumors of the infraobital nerve.
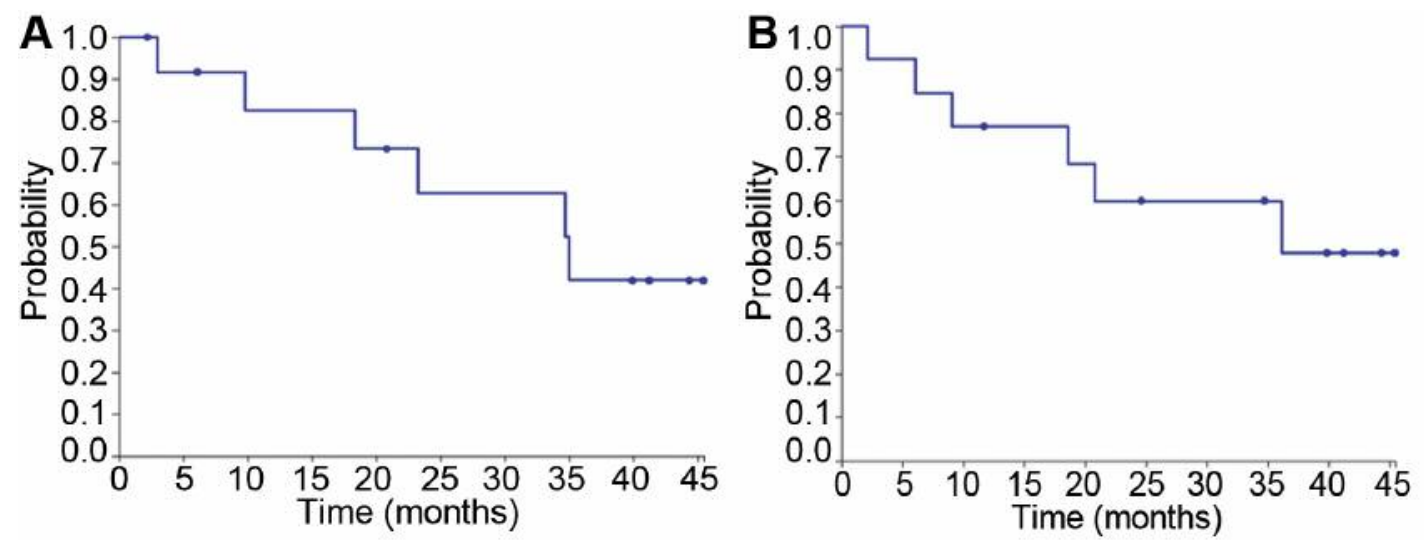

Figure 3. Local control (A) and overall survival (B) (entire cohort) after carbon ion radiotherapy.

Positive margins, even if microscopic, recurrence, and non-extremity site are strongly associated with significantly increased rate of local recurrence and some authors suggest additional RT with high doses in order to overcome radioresistance $(3-6,9,10)$.

In pediatric MPNST, complete surgical removal is also a strong predictor of survival, but RT showed a trend to be associated with better LC, especially when there is a minimal residual tumor (11).
Bishop et al. retrospectively analyzed 71 consecutive patients with MPNST who underwent surgery with neoadjuvant ( $32 \%$ of patients, median dose of $50 \mathrm{~Gy}$ ) or adjuvant (68\%, median dose of $64 \mathrm{Gy)}$ RT, reporting 5-year LC rates of $84 \%$ and low a toxicity profile. In their study, surgical margin status was the only factor associated with local relapse at 5 years $(28 \% v s .5 \%$ for those with negative margins; $p=0.02$; hazard ratio $=5.92,95 \%$ confidence interval=1.3-27.4) (12). 
In this retrospective study, we report the results of our series of MPNSTs treated with CIRT at the National Center of Oncologic Hadrontherapy (CNAO), which, in our opinion, can provide additional information for the clinical management of this aggressive disease.

At CNAO, heavy-ion radiotherapy is used for treatment of recurrence and in cases of positive margins after surgery. Looking at literature, we found two studies analyzing CIRT results for patients with MPNST suffering from gross residual or unresectable disease. The first study was conducted at the Particle facility in Heidelberg in Germany. After a median follow-up of 17 months (range=3-31 months), three out of the 11 patients had experienced local progression. The actuarial 1- and 2-year LC rates were both $65 \%$ with mild toxicity.

In a report from the Working Group for Carbon Ion Radiotherapy for Bone and Soft Tissue Sarcomas for the National Institute of Radiological Sciences in Japan, data of 128 inoperable soft-tissue sarcomas treated with curative intent with exclusive CIRT are described (13). In this series, 15 patients had MPNST and 5-year LC was 52\%. Patients were treated with a 16-fraction schedule with four fractions per week over 4 weeks. The prescribed dose ranged from 64 to $73.6 \mathrm{~Gy}$ (RBE).

In agreement with previous results, in our experience CIRT yields very promising LC with low morbidity. However, Jensen et al. used a different approach, compared to our treatment modality, combining photons and CIRT (three patients) or CIRT only (eight patients). The median cumulative dose delivered was $60 \mathrm{~Gy}$ (RBE): all patients who underwent combined treatment received $50 \mathrm{~Gy}$ photon intensity-modulated RT in 25 fractions and 24 Gy RBE CIRT in eight fractions for a total cumulative dose of 74 Gy (RBE) corresponding to $80 \mathrm{~Gy}$ [biological effective dose (BED)]; patients receiving CIRT only were treated to a total median dose of 60 Gy RBE (range=60-66 Gy RBE) delivered in 2022 fractions (3 Gy RBE/fraction); patients already irradiated were re-irradiated with 54-60 Gy RBE in 18-20 fractions (5).

The dose applied in our series was instead based on the NIRS schedule after rescaling to correct for the different RBE model employed $(14,15)$.

In our opinion, despite the limitations of this study (namely the retrospective nature of the data, the small cohort and the short follow-up) and considering the impact on clinical outcomes in terms of LC and toxicity, CIRT is a promising, effective and safe treatment for MPNSTs. In contrast to photons, heavy-ions have superior physical and radiobiological characteristics, providing a potential tumoricidal effect on hypoxic, radioresistant and deeply located tumors. Indeed, carbon ions travel through tissue with a minimal dose deposition along their path and allow better sparing of normal tissue since the dose rapidly falls off beyond the Bragg peak $(5,12-16)$.

\section{Conclusion}

MPNST is an aggressive tumor with a particularly high rate of local recurrence, and complete surgical resection remains the mainstay of a successful treatment. However, complete surgical excision at diagnosis is rarely feasible and even if re-excision is recommended, adjuvant RT can improve LC after incomplete surgery. Considering the radiobiological and physical features, CIRT may have a potential benefit in terms of LC and low morbidity. Our results suggest that CIRT may be effective, safe and well-tolerated, making it a promising approach in the management of MPNSTs.

Longer follow-up is needed to better investigate long-term results in terms of control and potential side-effects.

\section{Conflicts of Interest}

The Authors report no conflict of interest in regard to this study.

\section{References}

1 Grobmyer S, Reith JD, Shahlaee A, Bush CH and Hochwald SN: Malignant peripheral nerve sheath tumor: molecular pathogenesis and current management considerations. J Surg Oncol 97: 340349, 2008.

2 Goertz O, Langer S, Uthoff D, Ring A, Stricker I, Tannapfel A and Steinau HU: Diagnosis, treatment and survival of 65 patients with malignant peripheral nerve sheath tumors. Anticancer Res 34: 777-783, 2014.

3 Wong WW, Hirose T, Scheithauer BW, Schild SE and Gunderson LL: Malignant peripheral nerve sheath tumor: Analysis of treatment outcome. Int J Radiat Oncol Biol Phys 42: 351-360, 1998.

4 Anghileri M, Miceli R, Fiore M, Mariani L, Ferrari A, Mussi C, Lozza L, Collini P, Olmi P, Casali PG, Pilotti S and Gronchi A: Malignant peripheral nerve sheath tumors: Prognostic factors and survival in a series of patients treated at a single institution. Cancer 107: 1065-1074, 2006.

5 Jensen AD, Uhl M, Chaudhri N, Herfarth KK, Debus J and Roeder F: Carbon Ion irradiation in the treatment of grossly incomplete or unresectable malignant peripheral nerve sheaths tumors: acute toxicity and preliminary outcome. Radiat Oncol 10: 109, 2015.

6 Kepka L, DeLaney TF, Suit HD and Goldberg SI: Results of radiation therapy for unresected soft-tissue sarcomas. Int $\mathrm{J}$ Radiat Oncol Biol Phys 63: 852-859, 2005.

7 Uhl M, Mattke M, Welzel T, Roeder F, Oelmann J, Habl G, Jensen A, Ellerbrock, M, Jäkel O, Haberer T, Herfarth K and Debus J: Highly effective treatment of skull base chordoma with carbon ion irradiation using a raster scan technique in 155 patients: first long-terms results. Cancer 120: 3410-347, 2014.

8 National Cancer Institute: Common Terminology Criteria for Adverse Events (CTCAE), Version 4.0. Available at: http:// evs.nci.nih.gov/ftp1/CTCAE/About.html. Accessed on 27 September 2018.

9 Roeder F, Lehner B, Schmitt T, Kasper B, Egerer G, Sedlaczek O, Grüllich C, Mechtersheimer G, Wuchter P, Hensley FW, Huber PE, Debus J and Bischof M: Excellent local control with 
IOERT and postoperative EBRT in high-grade extremity sarcoma: Results from a subgroup analysis of a prospective trial. BMC Cancer 14: 350, 2014

10 Loree TR, North JH, Werness BA, Nangia R, Mullins AP and Hicks WL: Malignant peripheral nerve sheath tumors of the head and neck: Analysis of prognostic factors. Otolarygol Head Neck Surg 122: 667-672, 2000.

11 Carli M, Ferrari A, Mattke A, Zanetti I, Casanova M, Bisogno G, Cecchetto G, Alaggio R, De Sio L, Koscielniak E, Sotti G and Treuner J: Pediatric malignant peripheral nerve sheath tumor: The Italian and German soft-tissue sarcoma cooperative group. J Clin Oncol 23: 8422-8433, 2005.

12 Bishop AJ, Zagars GK, Torres KE, Bird JE, Feig BW and Guadagnolo BA: Malignant peripheral nerve sheath tumors: A single institution's experience using combined surgery and radiation therapy. Am J Clin Oncol 41(5): 465-470, 2018.

13 Imai R, Kamada T, Araki N and the Working Group for Carbon Ion Radiotherapy for Bone and Soft Tissue Sarcomas: Carbon ion radiotherapy for unresectable localized axial soft tissue sarcoma. Cancer Med 7(9): 4308-4314, 2018.
14 Fossati P, Molinelli S, Matsufuji N, Ciocca M, Mirandola A, Mairani A, Mizoe J, Hasegawa A, Imai R, Kamada T, Orecchia $\mathrm{R}$ and Tsujii $\mathrm{H}$ : Dose prescription in carbon ion radiotherapy: a planning study to compare NIRS and LEM approaches with a clinically-oriented strategy. Phys Med Biol 57: 7543-7554, 2012.

15 Molinelli S, Magro G, Mairani A, Matsufuji N, Kanematsu N, Inaniwa T, Mirandola A, Russo S, Mastella E, Hasegawa A, Tsuji H, Yamada S, Vischioni B, Vitolo V, Ferrari A, Ciocca M, Kamada T, Tsujii H, Orecchia R and Fossati P: Dose prescription in carbon ion radiotherapy: How to compare two different RBEweighted dose calculation systems. Radiother Oncol 120: 307$312,2016$.

16 Mitin T and Zietman AL: Promise and pitfalls of heavy-particle therapy. J Clin Oncol 32: 2855-2863, 2014.

Received December 7, 2018

Revised December 13, 2018

Accepted December 17, 2018 